\title{
THE EQUIVALENCE OF PAIRS OF HERMITIAN MATRICES*
}

\author{
BY \\ M. H. INGRAHAM AND K. W. WEGNER
}

Two pairs of $n$-ary Hermitian forms with $n \times n$ matrices $A, B$ and $C, D$ with elements in the complex field are equivalent if there exists a nonsingular matrix $T$ such that $\bar{T}^{\prime} A T=C$ and $\bar{T}^{\prime} B T=D$, where $\bar{T}^{\prime}$ is the conjugate-transpose of $T$.

As is usual in the study of equivalence of pairs of matrices the work divides itself into the consideration of the non-singular and singular cases. These two cases are taken up in Parts II and I respectively.

In the non-singular case the rank of $\rho A+\sigma B$ is $n$ except for special values of $\rho$ and $\sigma$. It has frequently been pointed out that in this case no generality is lost by assuming $B$ is of rank $n$.

In the singular case the rank $r$ of $\rho A+\sigma B$ is less than $n$ for all values of $\rho$ and $\sigma$, but as above no generality is lost in assuming that the rank $r$ of $B$ is the maximum rank of $\rho A+\sigma B$.

By the elementary divisors of a pair of matrices $A, B$ is meant the elementary divisors of $A-\lambda B$ when $B$ is non-singular, and the elementary divisors of $\rho A+\sigma B$ when $B$ is singular but the determinant $|\rho A+\sigma B|$ is not identically zero in $\rho$ and $\sigma$. In the non-singular case, the well known necessary and sufficient condition for the equivalence in any field of pairs of bilinear forms, or of their corresponding matrices, and for the equivalence in the field of complex numbers of pairs of symmetric matrices is that the pairs have the same elementary divisors. This condition is known to be not sufficient

* Presented to the Society, April 7, 1934; received by the editors June 27, 1934. Part II is in essence a dissertation of Dr. Wegner, written at the University of Wisconsin. Part I is in general the work of Professor Ingraham.

The subject of this paper has been the source of a considerable amount of current investigation some of which has led to results which in part are equivalent to some of those arrived at here. Dr. Wegner presented a paper containing his results at the April, 1934, meeting of the Society, the abstract appearing in the Bulletin of the American Mathematical Society, vol. 40, No. 1, January, 1934, as abstract No. 103. Simultaneously Dr. G. R. Trott obtained by somewhat analogous methods results which were equivalent to those of Dr. Wegner. These along with the proof of their equivalence are given in the American Journal of Mathematics, vol. 56, July, 1934, pp. 359 ff.: On the canonical form of a non-singular pencil of Hermitian matrices. Since preparing this paper it has been brought to the attention of the authors that Professor Turnbull had considered this problem and would soon publish a paper on the subject. The authors immediately submitted a copy of this paper to Professor Turnbull and in reply received word that the treatments were totally different, his treatment following the analogous classical treatment for the case of real quadratic forms. 
for the equivalence in the field of real numbers of pairs of real symmetric matrices. This is illustrated by the pairs of one-by-one matrices

$$
A=(1), B=(1) ; C=(-1), D=(-1)
$$

which have the same elementary divisor $(\lambda-1)$, but for which there obviously exists no real $P=(p)$ such that $P^{\prime} A P=p^{2}=-1$. In $1905 \mathrm{Muth}^{*}$ gave the necessary and sufficient conditions for the real equivalence of real symmetric matrices.

It has sometimes been stated that for the non-singular case the coincidence of the elementary divisors of the pairs is also a sufficient condition for the equivalence in the complex field of pairs of Hermitian matrices. That this is not the case is illustrated by the above pair considered as Hermitian matrices, there existing no $P=(p)$ such that $\bar{P}^{\prime} A P=\bar{p} p=-1$. The present paper gives the necessary and sufficient conditions for the equivalence of pairs of Hermitian matrices. Although the method of proof is much simpler, the conditions for the non-singular case are the same as those arrived at by Muth for the real symmetric case, a result which is entirely reasonable when one considers that Hermitian matrices should be thought of as a generalization of real symmetric matrices. Also, when one remembers that the necessary and sufficient conditions for the real equivalence of two real symmetric matrices or for the equivalence in the complex field of two Hermitian matrices is that they have the same rank and the same index, the results of Part II of this paper seem quite reasonable when stated in the following form:

TheOREm. Two pairs of Hermitian matrices $A, B$ and $C, D$, where $|B| \neq 0$ and $|D| \neq 0$, are equivalent if and only if

(1) they have the same elementary divisors, and

(2) the matrices $B\left(B^{-1} A-\lambda I\right)^{n}$ and $D\left(D^{-1} C-\lambda I\right)^{n}$ have the same index for all positive integral $n$ and real $\lambda$.

Dickson's $\dagger$ treatment of the singular case is reduced to the above mentioned erroneous treatment of the non-singular case. A direct reduction to the non-singular case, leading to a canonical form and using the Hermitian properties of the matrices involved, has been found. This is given in Part I. Part II, which treats the non-singular case, may be read independently of Part I.

* Muth, P., Über reelle Äquivalenz von Scharen reeller quadratischer Formen, in Journal für die reine und angewandte Mathematik, vol. 128 (1905), pp. 302-321.

$\dagger$ Dickson, L. E., Singular case of pairs of bilinear, quadratic, or Hermitian forms, these Transactions, vol. 29 (1927), pp. 239-253. 


\section{Singular CASE}

In this section the finding of a canonical form for a pair $A, B$ of Hermitian matrices is reduced to the treatment of a pair of lower order. By successive reductions the problem is completely solved or is finally reduced to the treatment of the non-singular case.

Consider a pair of $n \times n$ Hermitian matrices $A, B$ such that the rank of $\rho A+\sigma B$ never exceeds $r$, the rank of $B$. Without loss of generality we may assume that $B$ is of the form

$$
\left\|\begin{array}{ll}
B_{11} & 0 \\
0 & 0
\end{array}\right\| \text { where } B_{11}=\left\|\begin{array}{lr}
I_{s} & 0 \\
0 & -I_{t}
\end{array}\right\|,
$$

$I_{k}$ is the $k \times k$ identity matrix, the 0 's stand for 0 matrices, and $r=s+t$.

Let

$$
A=\left\|\begin{array}{ll}
A_{11} & A_{12} \\
\bar{A}_{12}^{\prime} & A_{22}
\end{array}\right\|,
$$

where $A_{11}$ is an $r \times r$ Hermitian matrix, $A_{12}$ is an $r \times(n-r)$ matrix, and $A_{22}$ is an $(n-r) \times(n-r)$ Hermitian matrix. Since the rank of $A+\sigma B$ never exceeds $r, A_{22}=0$, for if that were not the case there would be a minor of order $r+1$ the determinant of which would have $\pm k \sigma^{r}$ for the leading term in $\sigma$, where $k$ is a non-zero element of $A_{22}$, and hence this determinant is not identically zero.

Thus

$$
A+\sigma B=\left\|\begin{array}{lc}
A_{11}+\sigma B_{11} & A_{12} \\
\bar{A}_{12}^{\prime} & 0
\end{array}\right\|,
$$

where $A_{11}+\sigma B_{11}$ is non-singular, i.e., of rank $r$ except for a finite number of values of $\sigma$, and the rank of $A+\sigma B$ never exceeds $r$. Clearly,

$$
\left(A_{11}+\sigma B_{11}\right)\left(A_{11}+\sigma B_{11}\right)^{-1} A_{12}=A_{12}
$$

and from above the same relation must hold between the columns of the last $n-r$ rows of $A+\sigma B$ and hence

$$
\bar{A}_{12}^{\prime}\left(A_{11}+\sigma B_{11}\right)^{-1} A_{12}=0 .
$$

Since $B_{11}=B_{11}^{-1}$, for sufficiently large values of $\sigma$ we have the expansion

$$
\left(A_{11}+\sigma B_{11}\right)^{-1}=\frac{1}{\sigma} B_{11}-\frac{1}{\sigma^{2}} B_{11} A_{11} B_{11}+\frac{1}{\sigma^{3}} B_{11}\left(A_{11} B_{11}\right)^{2}+\cdots,
$$

and therefore from equation (2) we see that

$$
\bar{A}_{12}^{\prime} B_{11} A_{12}=0
$$


and in general

$$
\bar{A}_{12}^{\prime} B_{11}\left(A_{11} B_{11}\right)^{k} A_{12}=0 \quad(k=1,2,3, \cdots)
$$

If we let

$$
A_{12}=\left\|\begin{array}{l}
A_{121} \\
A_{122}
\end{array}\right\|
$$

where $A_{121}$ is an $s \times(n-r)$ matrix and $A_{122}$ is a $t \times(n-r)$ matrix, condition (3) becomes

$$
\bar{A}_{121}^{\prime} A_{121}=\bar{A}_{122}^{\prime} A_{122},
$$

and hence the ranks of $A_{121}$ and $A_{122}$ can not exceed the smallest of the three numbers $s, t$, and $n-r$.

We shall specify that in our canonical form for the pair $A, B, B$ as defined in equation (1) be left invariant.

Let $T$ be a non-singular matrix

$$
\left\|\begin{array}{ll}
T_{11} & T_{12} \\
T_{21} & T_{22}
\end{array}\right\|
$$

with the same conventions as above for the dimensions of the sub-matrices and satisfying the condition

$$
\bar{T}^{\prime} B T=B
$$

This condition is equivalent to the following three conditions:

$$
\bar{T}_{11}^{\prime} B_{11} T_{11}=B_{11}, \quad \bar{T}_{11}^{\prime} B_{11} T_{12}=0, \quad \bar{T}_{12}^{\prime} B_{11} T_{12}=0 .
$$

From the first of these conditions it follows that $T_{11}$ must be non-singular and hence from the second condition we see that $T_{12}=0$. Since $T$ is nonsingular, $T_{22}$ must be non-singular.

If $F=\bar{T}^{\prime} A T$, we have, using the same conventions as above,

$$
\begin{aligned}
& F_{11}=\bar{T}_{11}^{\prime} A_{11} T_{11}+\bar{T}_{21}^{\prime} \bar{A}_{12}^{\prime} T_{11}+\bar{T}_{11}^{\prime} A_{12} T_{21}, \\
& F_{12}=\bar{T}_{11}^{\prime} A_{12} T_{22}=\bar{F}_{21}^{\prime}, \\
& F_{22}=0 .
\end{aligned}
$$

As a special case we may take

$$
T_{11}=\left\|\begin{array}{ll}
S_{11} & 0 \\
0 & S_{22}
\end{array}\right\|
$$


where $S_{11}$ is an $s \times s$ unitary orthogonal matrix and $S_{22}$ is a $t \times t$ unitary orthogonal matrix, and for this case

$$
F_{121}=\bar{S}_{11}^{\prime} A_{121} T_{22}, \text { and } F_{122}=\bar{S}_{22}^{\prime} A_{122} T_{22} .
$$

Let the rank of $A_{121}$ be $l_{1}$. It is readily shown that, by a proper choice of $S_{11}$, $\bar{S}_{11}^{\prime} A_{121}$ may be taken as a matrix in which all the elements below the $l_{1}$ th row are zero. This being the case, it is readily seen that $T_{22}$ may be so chosen that

$$
F_{121}=\left\|\begin{array}{ll}
I_{l_{1}} & 0 \\
0 & 0
\end{array}\right\| .
$$

Although $T_{22}$ may have been determined, $S_{22}$ may be so chosen that all but the last $l$ rows of $F_{122}$ are zero, where $l$ is the rank of $A_{122}$, and such that in these last $l$ rows, if for the $i$ th and $(i+1)$ st rows $a_{i j}$ and $a_{i+1, k}$ are the first non-zero elements, then $j<k$. We may also pick $S_{22}$ such that these first nonzero elements are positive real numbers. From these conditions and the fact that according to (5)

$$
\bar{F}_{122}^{\prime} F_{122}=\bar{F}_{121}^{\prime} F_{121}
$$

we see that

$$
F_{122}=\left\|\begin{array}{ll}
0 & 0 \\
I_{l_{1}} & 0
\end{array}\right\|
$$

and that $l_{1}$ is the rank of $A_{12}$. In order not to accumulate notations we will assume from now on that

$$
A_{12}=\left\|\begin{array}{ll}
I_{l_{1}} & 0 \\
0 & 0 \\
I_{l_{1}} & 0
\end{array}\right\|,
$$

where, of course, if $l_{1}=n-r$ the second column of zeros is absent. Call $l_{1}$ the first invariant sub-rank of $A$.

We will now use only such transformations $T$ as will leave $B$ and the $A_{12}$ invariant. Let

and

$$
T_{11}=S
$$

$$
S=\left(S_{i j}\right) \quad(i=1,2,3 ; j=1,2,3),
$$

$S_{11}$ and $S_{33}$ being $l_{1} \times l_{1}$ matrices and $S_{22}$ an $\left(r-2 l_{1}\right) \times\left(r-2 l_{1}\right)$ matrix except where $r-2 l_{1}=0$, in which case the second row and column of $S$ are deleted. Let 


$$
B_{11}=\left\|\begin{array}{ccr}
I_{l_{1}} & 0 & 0 \\
0 & B_{11}^{(1)} & 0 \\
0 & 0 & -I_{l_{1}}
\end{array}\right\|,
$$

where $B_{11}^{(1)}$ is an $\left(r-2 l_{1}\right) \times\left(r-2 l_{1}\right)$ matrix of structure similar to $B_{11}$ with $s-l_{1}$ plus ones and $t-l_{1}$ minus ones on the main diagonal and elsewhere zero. From (9), we see that $\bar{S}^{\prime} A_{12} T_{22}=A_{12}$, and from this and the fact that $T_{22}$ is non-singular, it follows that

$$
\bar{S}^{\prime} A_{12}=\left\|\begin{array}{ll}
\bar{K}^{\prime} & 0 \\
0 & 0 \\
\bar{K}^{\prime} & 0
\end{array}\right\|,
$$

where $K$ is an $l_{1} \times l_{1}$ non-singular matrix. Hence, remembering the form (11) for $A_{12}$ we see that

$$
\begin{aligned}
& S_{31}=K-S_{11}, \\
& S_{13}=K-S_{33}, \\
& S_{32}=-S_{12} .
\end{aligned}
$$

Making these substitutions in the form for $S$, we see that the conditions that $\bar{S}^{\prime} B_{11} S=B_{11}$ are

$$
\begin{aligned}
& \bar{S}_{21}^{\prime} B_{11}^{(1)} S_{21}+\bar{S}_{11}^{\prime} K-\bar{K}^{\prime} K+\bar{K}^{\prime} S_{11}=I_{l_{1}}, \\
& \bar{S}_{21}^{\prime} B_{11}^{(1)} S_{22}+\bar{K}^{\prime} S_{12}=0 \\
& \bar{S}_{11}^{\prime} K+\bar{S}_{21}^{\prime} B_{11}^{(1)} S_{23}-\bar{K}^{\prime} S_{33}=0, \\
& \bar{S}_{22}^{\prime} B_{11}^{(1)} S_{22}=B_{11}^{(1)}, \\
& \bar{S}_{12}^{\prime} K+\bar{S}_{22}^{\prime} B_{11}^{(1)} S_{23}=0, \\
& \bar{K}^{\prime} K-\bar{K}^{\prime} S_{33}-\bar{S}_{33}^{\prime} K+\bar{S}_{23}^{\prime} B_{11}^{(1)} S_{23}=-I_{l_{1}} .
\end{aligned}
$$

Subtracting the conjugate-transpose of (16) from (19) we get

$$
\bar{S}_{22}^{\prime} B_{11}^{(1)}\left(S_{23}-S_{21}\right)=0
$$

and since $B_{11}^{(1)}$ is non-singular and, by (18), $S_{22}$ is non-singular, we see that

$$
S_{21}=S_{23} \text {. }
$$

Since $K$ is non-singular, from (16) we see that

$$
S_{12}=-\bar{K}^{\prime-1} \bar{S}_{21}^{\prime} B_{11}^{(1)} S_{22} \text {. }
$$

Using (21) and subtracting (17) from (15) we see that

$$
\bar{K}^{\prime} S_{11}+\bar{K}^{\prime} S_{33}-\bar{K}^{\prime} K=I_{l_{1}},
$$


that is,

$$
S_{11}+S_{33}-K=\bar{K}^{\prime-1} \text {. }
$$

Since subtracting (17) from (20) also yields (23) we see that conditions (17), (18), (21), (22) and (23) are equivalent to conditions (15) to (20). Moreover, it may be seen that $S_{21}$ may be chosen arbitrarily, that $S_{22}$ need only satisfy (18), and that $S_{11}$ and $S_{33}$ may be determined so as to satisfy (17) and (23).

Let us now turn our attention to equation (8) for $F_{11}$ and, in particular, study the last two terms $\bar{T}_{21}^{\prime} \bar{A}_{12}^{\prime} T_{11}+\bar{T}_{11}^{\prime} A_{12} T_{21}$ which we will call $M$.

If

we see that

$$
T_{21}=\left\|\begin{array}{lll}
T_{211} & T_{212} & T_{213} \\
T_{214} & T_{215} & T_{216}
\end{array}\right\|,
$$

$$
M=\left\|\begin{array}{lcc}
\bar{K}^{\prime} T_{211}+\bar{T}_{211}^{\prime} K & \bar{K}^{\prime} T_{212} & \bar{K}^{\prime} T_{213}+\bar{T}_{211}^{\prime} K \\
\bar{T}_{212}^{\prime} K & 0 & \bar{T}_{212}^{\prime} K \\
\bar{K}^{\prime} T_{211}+\bar{T}_{213}^{\prime} K & \bar{K}^{\prime} T_{212} & \bar{K}^{\prime} T_{213}+\bar{T}_{213}^{\prime} K
\end{array}\right\|,
$$

and we see, since $K$ is non-singular, that $M_{11}=\bar{K}^{\prime} T_{211}+\bar{T}_{211}^{\prime} K$ may be taken as an arbitrary $l_{1} \times l_{1}$ Hermitian matrix and that $M_{12}=\bar{K}^{\prime} T_{212}$ and $M_{13}=\bar{K}^{\prime} T_{213}$ $+\bar{T}_{211}^{\prime} K$ may be chosen as arbitrary matrices of the correct dimensions; that $M_{21}=M_{23}=\bar{M}_{12}^{\prime}=\bar{M}_{2}^{\prime}$, and that $M_{33}=M_{13}+M_{31}-M_{11}$. If now we write

$$
A_{11}=G=\left\|\begin{array}{lll}
G_{11} & G_{12} & G_{13} \\
\bar{G}_{12}^{\prime} & G_{22} & G_{23} \\
\bar{G}_{13}^{\prime} & \bar{G}_{23}^{\prime} & G_{33}
\end{array}\right\|,
$$

we see that condition (4) with $k=1$, namely, that $\bar{A}_{12}^{\prime} B_{11} G B_{11} A_{22}=0$, reduces to

$$
G_{33}=G_{13}+\bar{G}_{13}^{\prime}-G_{11} \text {. }
$$

Hence $M$ may be so chosen that

will be of the form

$$
F_{11}=\bar{T}_{11}^{\prime} A_{11} T_{11}+M
$$

$$
\left\|\begin{array}{ccr}
0 & 0 & 0 \\
0 & A_{11}^{(1)} & A_{12}^{(1)} \\
0 & \overline{A_{12}^{(1)}}, & 0
\end{array}\right\|
$$

We may consider $A_{11}$ to be in this form from now on. We must now study transformations $T$ which leave $B$ and $B_{11}$ invariant and which leave $F_{11}$ in form (24). Letting $H=\bar{S}^{\prime} A_{11} S$, we see that 


$$
\begin{aligned}
& H_{12}=\bar{S}_{21}^{\prime} A_{11}^{(1)} S_{22}+\bar{K}^{\prime} \overline{A_{12}^{(1)}} S_{22}-\bar{S}_{11}^{\prime} A_{12}^{(1)} S_{22}-\bar{S}_{21}^{\prime} A_{12}^{(1)} S_{12} \\
& H_{22}=\bar{S}_{22}^{\prime} A_{11}^{(1)} S_{22}-\bar{S}_{12}^{\prime} \overline{A_{12}^{(1)}} ' S_{22}-\bar{S}_{22}^{\prime} A_{12}^{(1)} S_{12} \\
& H_{23}=\bar{S}_{22}^{\prime} A_{11}^{(1)} S_{21}-\bar{S}_{12}^{\prime} \overline{A_{12}^{(1)}} S_{21}+\bar{S}_{22}^{\prime} A_{12}^{(1)} S_{33}
\end{aligned}
$$

If $M$ is so chosen that

then

$$
H+M=\left\|\begin{array}{ccc}
0 & 0 & 0 \\
0 & F_{11}^{(1)} & F_{12}^{(1)} \\
0 & \overline{F_{12}^{(1)}}, & F_{22}^{(1)}
\end{array}\right\|,
$$

$$
F_{22}^{(1)}=0, \quad F_{11}^{(1)}=H_{22},
$$

and

$$
F_{12}^{(1)}=H_{23}-\bar{H}_{12}^{\prime}=\bar{S}_{22}^{\prime} A_{12}^{(1)}\left(S_{33}+S_{11}-K\right),
$$

and by (23),

$$
F_{12}^{(1)}=\bar{S}_{22}^{\prime} A_{12}^{(1)} \bar{K}^{\prime-1} .
$$

If in (26) and (27) we replace

$$
F_{11}^{(1)}, F_{12}^{(1)}, F_{22}^{(1)}, A_{11}^{(1)} \text {, and } A_{12}^{(1)} \text { by } F_{11}, F_{12}, F_{22}, A_{11} \text {, and } A_{12} \text {, }
$$

and replace

$$
S_{22} \text { by } T_{11}, S_{12} \text { by }-T_{21} \text {, and } \bar{K}^{\prime-1} \text { by } T_{22} \text {, }
$$

we arrive at conditions (8), (9) and (10), and if in addition $B_{11}^{(1)}$ be replaced by $B_{11},(18)$ becomes (7). $A^{(1)}+\sigma B$, which is equal to

$$
\left\|\begin{array}{cccll}
\sigma I_{l_{1}} & 0 & 0 & I_{l_{1}} & 0 \\
0 & A_{11}^{(1)}+\sigma B_{11}^{(1)} & A_{12}^{(1)} & 0 & 0 \\
0 & A_{12}^{(1))^{\prime}} & -\sigma I_{l_{1}} & I_{l_{1}} & 0 \\
I_{l_{1}} & 0 & I_{l_{1}} & 0 & 0 \\
0 & 0 & 0 & 0 & 0
\end{array}\right\| \text {, }
$$

has the same rank as

$$
\left\|\begin{array}{ccccc}
\sigma I_{l_{1}} & 0 & 0 & 0 & 0 \\
0 & A_{11}^{(1)}+\sigma B_{11}^{(1)} & A_{12}^{(1)} & 0 & 0 \\
0 & A_{12}^{(1) '} & 0 & 0 & 0 \\
0 & 0 & 0 & -I_{l_{1}} / \sigma & 0 \\
0 & 0 & 0 & 0 & 0
\end{array}\right\| \text {, }
$$


and hence, since $A^{(1)}+\sigma B$ must have the same rank as $B$ except for a finite number of values of $\sigma$, the matrix

$$
\left\|\begin{array}{lc}
A_{11}^{(1)}+\sigma B_{11}^{(1)} & A_{12}^{(1)} \\
A_{11}^{(1)^{\prime}} & 0
\end{array}\right\|
$$

must have the same rank as $B_{11}^{(1)}$. This completes the reduction of the problem to the consideration of a pair of matrices of order $r$. Let $l_{2}$ be the first invariant sub-rank for $A^{(1)}, B^{(1)}$, etc. Finally at some stage $l_{k}$ is zero and then $A_{12}^{(k-1)}$ is zero and (8) reduces to the consideration of the non-singular case of the reduction of $A^{(k)}, B^{(k)}$. This completes the proof of the sufficiency of the conditions of Theorem 1 . The necessity may be readily checked from the above considerations.

THEOREM 1. Two pairs of Hermitian matrices $A, B$ and $C, D$ where the rank of $B$ is maximal for $\rho A+\sigma B$ are equivalent if and only if

(1) $B$ is equivalent to $D$;

(2) the invariant sub-ranks are equal;

(3) the non-singular pair $A^{(k)}, B^{(k)}$ is equivalent to the pair $C^{(k)}, D^{(k)}$.

II. NON-SINGULAR CASE

1. Preliminary reduction of pair. Consider any pair of Hermitian matrices $A, B$ with complex elements and such that the determinant $|\rho A+\sigma B|$ is not identically zero in $\rho$ and $\sigma$. As stated above, no generality is lost in assuming that $|B| \neq 0$. Calling $G$ the classical canonical form (described below) of $B^{-1} A$, we know there exists a matrix $T$ such that

$$
G=T^{-1} B^{-1} A T=T^{-1} B^{-1} \bar{T}^{\prime-1} \bar{T}^{\prime} A T=B_{1}^{-1} A_{1},
$$

where $B_{1}=\bar{T}^{\prime} B T$ and $A_{1}=\bar{T}^{\prime} A T$. Therefore we lose no generality in allowing the pair $A, B$ to be such that $B^{-1} A=G$ is in canonical form.

We shall call a matrix whose elements are all zero except for square blocks along the main diagonal a diagonal block matrix. It shall be shown that there exists a diagonal block matrix $E$ determined by $G$ such that $E=\bar{E}^{\prime}=E^{-1}$ and such that $B$ must be of the form $E S_{1}$, where $S_{1}$ is a matrix commutative with the canonical form $G$. A canonical pair $A_{c}, B_{c}$ for $A, B$ will then be obtained by showing that it is always possible to find a non-singular matrix $S$ commutative with $G$ such that

$$
B_{c}=\bar{S}^{\prime} B S \text { and } A_{c}=\bar{S}^{\prime} A S=B_{c} G \text {. }
$$

Let the elementary divisors of $A-\lambda B$ be $\left(\lambda-\lambda_{i}\right)^{e_{i}}$. Call $J_{i}$ the square matrix of order $e_{i}$ having ones in the diagonal above the main diagonal and other- 
wise zeros. Call $J_{i}{ }^{0}$ the identity matrix of order $e_{i}$. We may then describe the canonical form $G$ as a diagonal block matrix having a block $\left(\lambda_{i} J_{i}{ }^{0}+J_{i}\right)$ corresponding to each elementary divisor $\left(\lambda-\lambda_{i}\right)^{e_{i}}$. We may assume that blocks of $G$ which correspond to conjugate imaginary pairs of elementary divisors are adjacent blocks. Call $E_{i}$ the square matrix of order $e_{i}$ with ones along its secondary diagonal and otherwise zeros, i.e., having elements $\left(c_{j k}\right)$ where $c_{j k}=1$ for $j+k=e_{i}+1$, and $c_{j k}=0$ for $j+k \neq e_{i}+1$. Define $E$ as a diagonal block matrix such that a block $\left(\lambda_{i} J_{i}{ }^{0}+J_{i}\right)$ of $C$ corresponds to a block $E_{i}$ of $E$ when $\lambda_{i}$ is real, and two blocks $\left(\lambda_{i} J_{i}^{0}+J_{i}\right)$ and $\left(\bar{\lambda}_{i} J_{i}{ }^{0}+J_{i}\right)$ of $G$ correspond to one block

$$
\left\|\begin{array}{ll}
0 & E_{i} \\
E_{i} & 0
\end{array}\right\|
$$

of $E$ when $\lambda_{i}$ is not real. (In this paper the symbol 0 used in this way represents a matrix all of whose elements are zero.) This $E$ is such that $E=\bar{E}^{\prime}=E^{-1}$ and $E G E=\bar{G}^{\prime}$, whence, since we are assuming $B^{-1} A=G$,

$$
A=\bar{A}^{\prime}=B G=\bar{G}^{\prime} B=E G E B,
$$

and therefore

$$
E B G=G E B \text {. }
$$

Hence $B$ must be of the form $E S_{1}$, where $S_{1}$ is a matrix commutative with $G$.

The form of any matrix $S$ commutative with the canonical form $G$ will now be described. To facilitate this description, we may assume that the blocks of $G$ are arranged so that those corresponding to elementary divisors involving the same root appear in non-increasing order with respect to size.

Call $I_{i k}, e_{i} \geqq e_{k}$, the $e_{i} \times e_{k}$ matrix made up of $J_{k}^{0}$ augmented below by $e_{i}-e_{k}$ rows of zeros. $S$ is a block matrix of the following form: To the block $\left(\lambda_{i} J_{i}{ }^{0}+J_{i}\right)$ of $G$ corresponds a block $S_{i i}\left(J_{i}\right)$ of $S$, where $S_{i i}$ is a polynomial with complex coefficients. When $\lambda_{i}=\lambda_{k}, e_{i} \geqq e_{k}$, there is also an $e_{i} \times e_{k}$ block of the form $I_{i k} S_{i k}\left(J_{k}\right)$ in the columns of $S_{k k}\left(J_{k}\right)$ and the rows of $S_{i i}\left(J_{i}\right)$, and an $e_{k} \times e_{i}$ block of the form $S_{k i}\left(J_{k}\right) E_{k} I_{i k}^{\prime} E_{i}$ in the rows of $S_{k k}\left(J_{k}\right)$ and the columns of $S_{i i}\left(J_{i}\right)$.

$B=E S$ will then be a block matrix of the following form: Considering first the blocks related to elementary divisors involving real roots we find that to the block $S_{i i}\left(J_{i}\right)$ of $S$ corresponds a block $E_{i} B_{i i}\left(J_{i}\right)$ of $B$, to the block $I_{i k} S_{i k}\left(J_{k}\right)$ of $S$ the block $E_{i} I_{i k} B_{i k}\left(J_{k}\right)$ of $B$, and to the block $S_{k i}\left(J_{k}\right) E_{k} I_{i k}^{\prime} E_{i}$ of $S$ the block $\bar{B}_{i k}\left(J_{k}^{\prime}\right) I_{i k}^{\prime} E_{i}$ of $B$. Considering then double blocks related to conjugate imaginary pairs of elementary divisors we find that to

$$
\left\|\begin{array}{cc}
S_{i i 1}\left(J_{i}\right) & 0 \\
0 & S_{i i 2}\left(J_{i}\right)
\end{array}\right\|
$$


of $S$ corresponds

of $B$; to

$$
\left\|\begin{array}{cc}
0 & E_{i} B_{i i 1}\left(J_{i}\right)
\end{array}\right\|
$$

of $S$ corresponds

$$
\left\|\begin{array}{cc}
I_{i k} S_{i k 1}\left(J_{k}\right) & 0 \\
0 & I_{i k} S_{i k 2}\left(J_{k}\right)
\end{array}\right\|
$$

of $B$; and to

$$
\| \begin{array}{cc}
0 & E_{i} I_{i k} B_{i k 2}\left(J_{k}\right)
\end{array} \mid
$$

$$
\left\|\begin{array}{cc}
S_{k i 1}\left(J_{k}\right) E_{k} I_{i k}^{\prime} E_{i} & 0 \\
0 & S_{k i 2}\left(J_{k}\right) E_{k} I_{i k}^{\prime} E_{i}
\end{array}\right\|
$$

of $S$ corresponds

of $B$.

$$
\left\|\begin{array}{cc}
0 & \bar{B}_{i k 1}\left(J_{k}^{\prime}\right) I_{i k}^{\prime} E_{i} \\
\bar{B}_{i k 2}\left(J_{k}^{\prime}\right) I_{i k}^{\prime} E_{i} & 0
\end{array}\right\|
$$

2. Reduction of pair to canonical form. The canonical pair $A_{c}, B_{c}$ that we shall obtain has the following form: $B_{c}$ is a diagonal block matrix with blocks of the same dimensions as those of $E$, a block $E_{i}$ of $E$ corresponding to a block $\epsilon_{i} E_{i}$ of $B_{c}$, where $\epsilon_{i}= \pm 1$, and a block

$$
\left\|\begin{array}{ll}
0 & E_{i} \\
E_{i} & 0
\end{array}\right\|
$$

of $E$ corresponding to a block

$$
\left\|\begin{array}{ll}
0 & E_{i} \\
E_{i} & 0
\end{array}\right\|
$$

of $B_{c} . A_{c}=B_{c} G$ is also a diagonal block matrix with blocks of the same dimensions as those of $E$, a block $E_{i}$ of $E$ corresponding to a block $\epsilon_{i} E_{i}\left(\lambda_{i} J_{i}^{0}+J_{i}\right)$ of $A_{c}$, and a block

$$
\left\|\begin{array}{ll}
0 & E_{i} \\
E_{i} & 0
\end{array}\right\|
$$

of $E$ corresponding to a block

of $A_{c}$.

$$
\left\|\begin{array}{cc}
0 & E_{i}\left(\bar{\lambda}_{i} J_{i}{ }^{0}+J_{i}\right) \\
E_{i}\left(\lambda_{i} J_{i}{ }^{0}+J_{i}\right) & 0
\end{array}\right\|
$$


Suppose the real elementary divisors $\left(\lambda-\lambda_{i}\right)^{e_{i}}$ of $A, B$ divide into $m$ classes of equal elementary divisors (i.e., involving the same root and the same exponent). For each class define a $\sigma$ as the sum of the $\epsilon$ 's corresponding to the elementary divisors of that class. It will be shown in $\$ 3$ that these $m \sigma_{i}$ are invariants of the pair $A, B$.

To reduce to this canonical pair we shall show that it is always possible to choose a non-singular matrix $S$ commutative with the canonical form $G=B^{-1} A$ so that $\bar{S}^{\prime} B S=B_{c}$. It follows that

$$
A_{c}=\bar{S}^{\prime} A S=\bar{S}^{\prime} B S S^{-1} G S=\bar{S}^{\prime} B S G=B_{c} G .
$$

Because of the block form of $B$ and $S$ it is evidently necessary to consider elementary divisors involving but a single real root, or a pair of conjugate imaginary roots. The reduction is divided into eight cases. In Case I the canonical form is obtained for a pair of matrices having but one real elementary divisor, and in Case II for a pair of matrices having but one pair of conjugate imaginary elementary divisors. Every other situation is shown to depend essentially on Cases I and II. In Cases III and IV induction is used to obtain the canonical forms where there are any number of distinct (i.e., with distinct exponents, but involving the same characteristic root) and no repeated, elementary divisors (III) and any number of distinct, but no repeated, pairs of conjugate imaginary elementary divisors (IV). In Cases V and VI the situations of a cluster of equal real elementary divisors and a cluster of equal pairs of conjugate imaginary elementary divisors are reduced so as to be handled by the methods of Cases III and IV. Finally, in Cases VII and VIII, induction on Cases V and VI is used to cover the situation of any number of clusters of equal real elementary divisors and of any number of clusters of equal pairs of conjugate imaginary elementary divisors.

Before taking up these cases, it will be well to list some relations that shall be used repeatedly in the reductions:

$$
\begin{aligned}
J_{i}^{j} & =0 & & \text { when } j \geqq e_{i} ; \\
P\left(J_{i}^{\prime}\right) E_{i} & =E_{i} P\left(J_{i}\right), & & P \text { a polynomial } ; \\
P_{1}\left(J_{j}\right) I_{j k} & =I_{j k} P_{2}\left(J_{k}\right), & & P_{1} \text { and } P_{2} \text { polynomials } ; \\
E_{k} I_{j k}^{\prime} E_{j} I_{j k} & =J_{k}^{e j-e k} ; & & \\
I_{i j} I_{j k} & =I_{i k} . & &
\end{aligned}
$$

Also, it will be found convenient in some of the reductions to use for references the following multiplications, in which $\mathcal{S}_{i j}, \mathcal{B}_{i j}$, and $\mathbb{R}_{i j}$ are themselves matrices, square if $i=j$.

If 
then

$$
S=\left\|\begin{array}{cc}
S_{11} & S_{12} \\
0 & I
\end{array}\right\| \text { and } B=\left\|\begin{array}{cc}
\mathcal{B}_{11} & \mathcal{B}_{12} \\
\bar{B}_{12}^{\prime} & \mathcal{B}_{22}
\end{array}\right\|
$$

(7) $\quad \bar{S}^{\prime} B S=\left\|\begin{array}{ll}\bar{S}_{11}^{\prime} \mathcal{B}_{11} S_{11} & \bar{S}_{11}^{\prime}\left(B_{1} S_{12}+B_{12}\right) \\ \left.\overline{(S}_{12}^{\prime} \bar{B}_{11}^{\prime}+\bar{B}_{12}^{\prime}\right) S_{11} & \bar{S}_{12}^{\prime}\left(\mathcal{B}_{11} S_{12}+\mathcal{B}_{12}\right)+\bar{B}_{12}^{\prime} S_{12}+\mathcal{B}_{22}\end{array}\right\|$.

If

$$
R=\left\|\begin{array}{ll}
I & 0 \\
0 & R_{22}
\end{array}\right\| \text { and } B=\left\|\begin{array}{ll}
\mathcal{B}_{11} & \mathcal{B}_{12} \\
\overline{\mathcal{B}}_{12}^{\prime} & \mathcal{B}_{22}
\end{array}\right\|
$$

then

$$
\bar{R}^{\prime} B R=\left\|\begin{array}{cc}
\mathcal{B}_{11} & \mathcal{B}_{12} \\
\bar{B}_{12}^{\prime} & \bar{R}_{22}^{\prime} \mathcal{B}_{22} \mathcal{R}_{22}
\end{array}\right\|
$$

In some of the cases below, manipulative details have been omitted. Those interested may refer to the doctor's thesis, University of Wisconsin, 1934, by K. W. Wegner.

Case I. A single elementary divisor $\left(\lambda-\lambda_{1}\right)^{e_{1}}$.

$$
\begin{aligned}
S= & S_{1}\left(J_{1}\right)=\sum_{i=1}^{e_{1}} s_{i} J_{1}^{i-1}, \quad B=E_{1} B_{1}\left(J_{1}\right)=E_{1} \cdot \sum_{i=1}^{e 1} b_{i} J_{1}^{i-1} \\
\bar{S}^{\prime} B S= & \bar{S}_{1}\left(J_{1}^{\prime}\right) E_{1} B_{1}\left(J_{1}\right) S_{1}\left(J_{1}\right)=E_{1} \bar{S}_{1}\left(J_{1}\right) B_{1}\left(J_{1}\right) S_{1}\left(J_{1}\right) \quad \text { by }(3) \\
= & E_{1}\left[s_{1}^{2} b_{1} J_{1}^{0}+\left(2 s_{1} s_{2} b_{1}+s_{1}^{2} b_{2}\right) J_{1}+\left(2 s_{1} s_{3} b_{1}+s_{2}^{2} b_{1}+2 s_{1} s_{2} b_{2}+s_{1}^{2} b_{3}\right) J_{1}^{2}\right. \\
& +\left(2 s_{1} s_{4} b_{1}+2 s_{2} s_{3} b_{1}+2 s_{1} s_{3} b_{2}+s_{2}^{2} b_{2}+2 s_{1} s_{2} b_{3}+s_{1}^{2} b_{4}\right) J_{1}^{3}+\cdots \\
& \left.+\left(2 s_{1} s_{e_{1}} b_{1}+2 s_{2} s_{e_{1}-1} b_{1}+\cdots\right) J_{1}^{e_{1}-1}\right],
\end{aligned}
$$

using (2) and choosing the $s_{i}$ to be real. The element $b_{1}$ is real since $B$ is Hermitian and $b_{1} \neq 0$ since $|B| \neq 0$. Hence we may choose $s_{1}=\left( \pm b_{1}\right)^{-1 / 2}$ and $s_{j}, j=2,3, \cdots, e_{1}$, so that the coefficient of $J_{1}^{j-1}$ in the last expression for $\bar{S}^{\prime} B S$ above is zero. Then $\bar{S}^{\prime} B S=S^{\prime} B S= \pm E_{1} J_{1}^{0}= \pm E_{1}=\epsilon_{1} E_{1}=B_{c}$.

Case II. Elementary divisors: $\left(\lambda-\lambda_{1}\right)^{e_{1}},\left(\lambda-\bar{\lambda}_{1}\right)^{e_{1}}, \lambda_{1} \neq \bar{\lambda}_{1}$.

$$
\begin{aligned}
& S=\left\|\begin{array}{cc}
S_{1}\left(J_{1}\right) & 0 \\
0 & S_{2}\left(J_{1}\right)
\end{array}\right\|, \quad B=\left\|\begin{array}{cc}
0 & E_{1} B_{1}\left(J_{1}\right) \\
\bar{B}_{1}\left(J_{1}^{\prime}\right) E_{1} & 0
\end{array}\right\|, \\
& \bar{S}^{\prime} B S=\left\|\begin{array}{cc}
0 & \bar{S}_{1}\left(J_{1}^{\prime}\right) E_{1} B_{1}\left(J_{1}\right) S_{2}\left(J_{1}\right) \\
\bar{S}_{2}\left(J_{1}^{\prime}\right) \bar{B}_{1}\left(J_{1}^{\prime}\right) E_{1} S_{1}\left(J_{1}\right) & 0
\end{array}\right\| .
\end{aligned}
$$

Choose $S_{1}\left(J_{1}\right)=J_{1}^{0}$ and call

Then

$$
B_{1}\left(J_{1}\right)=\sum_{i=1}^{e_{1}} b_{i} J_{1}^{i-1}, \quad S_{2}\left(J_{1}\right)=\sum_{i=1}^{e_{1}} s_{i} J_{1}^{i-1} .
$$




$$
\begin{aligned}
\bar{S}_{1}\left(J_{1}^{\prime}\right) E_{1} B_{1}\left(J_{1}\right) S_{2}\left(J_{1}\right) & =E_{1}\left[s_{1} b_{1} J_{1}^{0}+\left(s_{2} b_{1}+s_{1} b_{2}\right) J_{1}+\left(s_{3} b_{1}+s_{2} b_{2}+s_{1} b_{3}\right) J_{1}^{2}\right. \\
& \left.+\cdots+\left(s_{e_{1}} b_{1}+s_{e_{1}-1} b_{2}+\cdots+s_{2} b_{e_{1}-1}+s_{1} b_{e_{1}}\right) J_{1}^{e_{1}-1}\right] .
\end{aligned}
$$

Since $|B| \neq 0$, we know $b_{1} \neq 0$. Hence we may choose $s_{1}=1 / b_{1}$ and $s_{j}, j=2$, $3, \cdots, e_{1}$, so that the coefficient of $J_{1}{ }^{i-1}$ in the above expression is zero. Then

$$
\bar{S}^{\prime} B S=\left\|\begin{array}{ll}
0 & E_{1} \\
E_{1} & 0
\end{array}\right\|=B_{c} .
$$

Case III. Elementary divisors:

$$
\left(\lambda-\lambda_{1}\right)^{e_{1}},\left(\lambda-\lambda_{1}\right)^{e_{2}},\left(\lambda-\lambda_{1}\right)^{e_{3}}, \cdots,\left(\lambda-\lambda_{1}\right)^{e_{k}}, e_{1}>e_{2}>\cdots>e_{k} .
$$

In (7) we may take

$S_{11}$ of the form described for $S$ in $\$ 1$ with $k-1$ diagonal blocks and $\frac{1}{2}(k-1)(k-2)$ blocks above the diagonal. The $\frac{1}{2}(k-1)(k-2)$ blocks below the diagonal are taken to be zero;

$S_{12}$ a matrix of dimensions $\left(e_{1}+e_{2}+\cdots+e_{k-1}\right) \times e_{k}$ made up of matrices, each above the next, of the form $I_{i k} S_{i k}\left(J_{k}\right), i=1,2, \cdots,(k-1)$;

$B_{11}$ of the form described for $B$ in $\$ 1$ with $k-1$ diagonal blocks and $\frac{1}{2}(k-1)(k-2)$ blocks above and also below the diagonal;

$B_{12}$ a matrix of dimensions $\left(e_{1}+e_{2}+\cdots+e_{k-1}\right) \times e_{k}$ made up of matrices, each above the next, of the form $E_{i} I_{i k} B_{i k}\left(J_{k}\right), i=1,2, \cdots,(k-1)$;

$\mathcal{B}_{22}=E_{k} B_{k k}\left(J_{k}\right)$.

We know that $\left|\mathcal{B}_{11}\right| \neq 0$ and $\left|\mathcal{B}_{22}\right| \neq 0$ since $|B| \neq 0$ and $e_{1}>e_{k}$. Let us assume that $S_{11}$ can be chosen so that $\bar{S}_{11}^{\prime} \mathcal{B}_{11} \mathcal{S}_{11}$ is of the desired form, i.e., a matrix of $k-1$ diagonal blocks of the form $\epsilon_{i} E_{i}$. (See Case I for start of induction.) Since $B_{11} \delta_{12}$ is of the same form as $\mathcal{B}_{12}$, we may choose $\delta_{12}$ so that $\mathcal{B}_{11} S_{12}+\mathcal{B}_{12}=0$.

$\overline{\mathcal{B}}_{12}^{\prime} S_{12}=\sum_{i=1}^{k-1} \bar{B}_{i k}\left(J_{k}^{\prime}\right) I_{i k}^{\prime} E_{i} I_{i k} S_{i k}\left(J_{k}\right)=\sum_{i=1}^{k-1} E_{k} \bar{B}_{i k}\left(J_{k}\right) J_{k}^{e_{i}-e_{k}} S_{i k}\left(J_{k}\right)=E_{k} P_{1}\left(J_{k}\right)$,

where $P_{1}$ has no constant term. Since $\mathcal{B}_{22}+E_{k} P_{1}\left(J_{k}\right)$ is therefore non-singular, we employ a further transformation $R$ of form (8) in which we may, according to Case $I$, choose $R_{22}$ so that

$$
\bar{R}_{22}^{\prime}\left[\mathcal{B}_{22}+E_{k} P_{1}\left(J_{k}\right)\right] R_{22}=\epsilon_{k} E_{k} .
$$

Calling $S_{1}=S R$, we have $\bar{S}_{1}^{\prime} B S_{1}=B_{c}$.

Evidently this method would cover the situation with $e_{1} \geqq e_{2} \geqq \cdots \geqq e_{k}$ if it were known that $\left|\mathcal{B}_{11}\right| \neq 0,\left|\mathcal{B}_{22}\right| \neq 0$, and that $\mathcal{B}_{12}$ involved only polynomials without constant terms. 
Case IV. Elementary divisors:

$$
\begin{array}{r}
\left(\lambda-\lambda_{1}\right)^{e_{1}},\left(\lambda-\bar{\lambda}_{1}\right)^{e_{1}},\left(\lambda-\lambda_{1}\right)^{e_{2}},\left(\lambda-\bar{\lambda}_{1}\right)^{e_{2}}, \cdots,\left(\lambda-\lambda_{1}\right)^{e_{k}},\left(\lambda-\bar{\lambda}_{1}\right)^{e_{k}}, \\
e_{1}>e_{2} \cdots>e_{k}, \lambda_{1} \neq \bar{\lambda}_{1} .
\end{array}
$$

This case may be handled by exactly the same method as that used in Case III, double blocks being dealt with in place of single ones. The note at the end of Case III is also valid here.

Case V. Elementary divisors:

$$
\left(\lambda-\lambda_{1}\right)^{e_{1}},\left(\lambda-\lambda_{1}\right)^{e_{2}}, \cdots,\left(\lambda-\lambda_{1}\right)^{e k}, \quad e_{1}=e_{2}=\cdots=e_{k} .
$$

$S$ contains $k^{2}$ blocks of form

$$
S_{i j}\left(J_{1}\right)=\sum_{n=1}^{e_{1}} s_{i j n} J_{1}^{n-1} \quad(i, j=1,2, \cdots, k) .
$$

$B$ contains $k^{2}$ blocks of form

$$
E_{1} B_{i j}\left(J_{1}\right)=E_{1} \sum_{n=1}^{e_{1}} s_{i j n} J_{1}^{n-1} \quad(i, j=1,2, \cdots, k) .
$$

Hence $\bar{S}^{\prime} B S$ contains $k^{2}$ blocks of form $E_{1} P_{i j}\left(J_{1}\right)$ in which the coefficients of $J_{1}^{0}$ in the polynomials $P_{i j}$ involve only $s_{i j n}$ and $b_{i j n}$ with $n=1$. Since

$$
\pm\left|\begin{array}{cccc}
b_{111} & b_{121} & \cdots & b_{1 k 1} \\
b_{121} & b_{221} & \cdots & b_{2 k 1} \\
\vdots & \vdots & \cdots & \vdots \\
\bar{b}_{1 k 1} & \bar{b}_{2 k 1} & \cdots & b_{k k 1}
\end{array}\right|^{e 1}=|B| \neq 0
$$

we may choose $s_{i j 1}$ so that

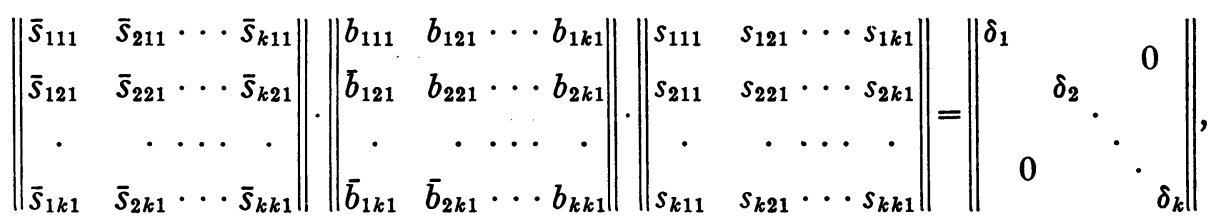

where $\delta_{i}= \pm 1$. Choosing $s_{i j n}=0$ for $n \neq 1$, we then apply the method of Case III to $\bar{S}^{\prime} B S=B_{1}$, the latter being such that $\left|\mathcal{B}_{11}\right| \neq 0,\left|\mathcal{B}_{22}\right| \neq 0$, and $\mathcal{B}_{12}$ involves polynomials in $J_{1}$ without constant terms. (See the remark at the end of Case III.)

Case VI. Elementary divisors:

$$
\begin{aligned}
\left(\lambda-\lambda_{1}\right)^{e_{1}},\left(\lambda-\bar{\lambda}_{1}\right)^{e_{1}},\left(\lambda-\lambda_{1}\right)^{e_{2}},\left(\lambda-\bar{\lambda}_{1}\right)^{e_{2}}, \cdots,\left(\lambda-\lambda_{1}\right)^{e_{k}},\left(\lambda-\bar{\lambda}_{1}\right)^{e_{k}} ; \\
e_{1}=e_{2}=\cdots=e_{k} ; \lambda_{1} \neq \bar{\lambda}_{1} .
\end{aligned}
$$


$S$ contains $k^{2}$ blocks of form

$$
\left|\begin{array}{cc}
S_{i 11}\left(J_{1}\right) & 0 \\
0 & S_{i j 2}\left(J_{1}\right)
\end{array}\right| \quad(i, j=1,2, \cdots, k),
$$

$B$ contains $k^{2}$ blocks of form

$$
\left|\begin{array}{cc}
0 & E_{1} B_{i j 2}\left(J_{1}\right) \\
E_{1} B_{i j 1}\left(J_{1}\right) & 0
\end{array}\right| \quad(i, j=1,2, \cdots, k)
$$

where

and

$$
S_{i j m}\left(J_{1}\right)=\sum_{n=1}^{e_{1}} s_{i j m n} J_{1}^{n-1} \quad(m=1,2),
$$

$$
B_{i j 2}\left(J_{1}\right)=\bar{B}_{j i 1}\left(J_{1}^{\prime}\right)=\sum_{n=1}^{e_{1}} b_{i j 2 n} J_{1}^{n-1} .
$$

Then $\bar{S}^{\prime} B S$ contains $k^{2}$ blocks of form

$$
\left\|\begin{array}{cc}
0 & E_{1} P_{i j 2}\left(J_{1}\right)
\end{array}\right\|_{E_{1} P_{i j 1}\left(J_{1}\right)}^{0} \quad 0 \quad(i, j=1,2, \cdots, k),
$$

in which the coefficients of $J_{1}{ }^{0}$ in the polynomials $P_{i j m}$ involve only $s_{i j m n}$ and $b_{i j m n}$ with $n=1$. Choose $s_{i j m n}=0, n \neq 1$. Using the fact that $|B| \neq 0$, it can be shown by a method similar to that used in Case $\mathrm{V}$ that we may choose $s_{i j 11}=s_{i j 21}$ in such a way that $\bar{S}^{\prime} B S=S_{1}$ can be handled by the method of Case IV. (See remark at end of Case IV.)

Case VII. Elementary divisors: $\left(\lambda-\lambda_{1}\right)^{i}$, and

Case VIII. Elementary divisors: $\left(\lambda-\lambda_{1}\right)^{i},\left(\lambda-\bar{\lambda}_{1}\right)^{j}, \lambda_{1} \neq \bar{\lambda}_{1}$, in which, for both cases, $j$ takes on the values $e_{1}, e_{2}, \cdots, e_{\beta_{k}}$, where

$$
\begin{aligned}
e_{1}=e_{2}=e_{3} & =\cdots=e_{\beta_{1}}>e_{\beta_{1}+1}=e_{\beta_{1}+2}=\cdots=e_{\beta_{2}}>e_{\beta_{2}+1} \\
& =\cdots=e_{\beta_{3}}>e_{\beta_{3+1}}=\cdots>e_{\beta_{k-1}+1}=\cdots=e_{\beta_{k}} .
\end{aligned}
$$

These cases may be handled as were Cases III and IV, they being made to depend on V and VI as III and IV depended on I and II. Clusters of blocks are dealt with in place of single blocks.

3. Conditions for equivalence. Consider the pair of Hermitian matrices $A, B$, where $|B| \neq 0$. The matrix $B\left(B^{-1} A-\lambda I\right)^{n}$ is Hermitian for any real $\lambda$ and positive integral $n$ since it is a sum of matrices of the form $B\left(B^{-1} A\right)^{i}$, which is easily shown to be Hermitian for any positive integral $j$.

Referring to the $\sigma_{i}$ defined at the beginning of $\$ 2$, the following theorem may be stated: 
THEOREM 2. In the non-singular case, two pairs of Hermitian matrices are equivalent if and only if they have the same elementary divisors and also the same $\sigma_{i}$.

We shall prove this theorem in a more illuminating form already stated above:

TheOREM 2a. Two pairs of Hermitian matrices $A, B$ and $C, D$, where $|B| \neq 0$ and $|D| \neq 0$, are equivalent if and only if

(1) they have the same elementary divisors, and

(2) the matrices $B\left(B^{-1} A-\lambda I\right)^{n}$ and $D\left(D^{-1} C-\lambda I\right)^{n}$ have the same index for all positive integral $n$ and real $\lambda$.

Necessity. The necessity of (1) is known from classical theory.

Suppose there exists a non-singular $P$ so that $\bar{P}^{\prime} A P=C$ and $\bar{P}^{\prime} B P=D$. Then

$$
\begin{aligned}
D\left(D^{-1} C-\lambda I\right)^{n} & =\bar{P}^{\prime} B P\left(P^{-1} B^{-1} \bar{P}^{\prime-1} \bar{P}^{\prime} A P-\lambda I\right)^{n} \\
& =\bar{P}^{\prime} B P\left[P^{-1}\left(B^{-1} A-\lambda I\right) P\right]^{n} \\
& =\bar{P}^{\prime} B P\left[P^{-1}\left(B^{-1} A-\lambda I\right)^{n} P\right] \\
& =\bar{P}^{\prime}\left[B\left(B^{-1} A-\lambda I\right)^{n}\right] P,
\end{aligned}
$$

whence the necessity of (2) follows.

Sufficiency. We may assume the two pairs are in their canonical forms. Because of (1), these canonical pairs are the same except possibly in their $\epsilon_{i}$. The coincidence of the $\sigma_{i}$ is a sufficient condition for equivalence since equal $\epsilon_{i}$ could be made to correspond by a proper interchange of blocks. We have then merely to prove that for any variation in the $\sigma_{i}$ of the canonical pairs there will exist a real $\lambda$ and a positive integral $n$ such that the indices of $B\left(B^{-1} A-\lambda I\right)^{n}$ and $D\left(D^{-1} C-\lambda I\right)^{n}$ are not the same.

Since $A$ and $B$ are in canonical form, $B\left(B^{-1} A-\lambda I\right)^{n}$ is a diagonal block matrix like $A$ and $B$, the real block $\epsilon_{i} E_{i}\left(\lambda_{i} J_{i}{ }^{0}+J_{i}\right)$ of $A$ corresponding to the block $\epsilon_{i} E_{i}\left[\left(\lambda_{i}-\lambda\right) J_{i}{ }^{0}+J_{i}\right]^{n}$ of $B\left(B^{-1} A-\lambda I\right)^{n}$. Obviously, the index of $B\left(B^{-1} A-\lambda I\right)^{n}$ will be the sum of the indices of its blocks.

Consider first the case in which all the elementary divisors involve the same real root $\lambda_{1}$, the exponents being

$$
\begin{aligned}
e_{1}=e_{2} & =\cdots=e_{\beta_{1}}>e_{\beta_{1+1}}=e_{\beta_{1}+2}=\cdots=e_{\beta_{2}}>e_{\beta_{2}+1} \\
& =\cdots=e_{\beta_{8}}>e_{\beta_{8+1}}=\cdots>\cdots=e_{\beta_{u}} .
\end{aligned}
$$

Let $e_{\beta_{s}}$ be the largest exponent such that 


$$
\sigma_{s}=\sum_{j=\beta_{s-1}+1}^{\beta_{s}} \epsilon_{j}
$$

of one pair is different from

$$
\sigma_{s}^{\prime}=\sum_{j=\beta_{s-1+1}}^{\beta_{s}} \epsilon_{j}^{\prime}
$$

of the other pair. Choose $\lambda=\lambda_{1}$ and $n=e_{\beta_{s}}-1$. Blocks $\epsilon_{i} E_{i} J_{i}{ }^{e \beta_{s}-1}$ will have the same indices in each pair when $i<\beta_{s}$, since we are assuming $\sigma_{j}=\sigma_{j}^{\prime}, j<s$. Also blocks $\epsilon_{i} E_{i} J_{i}{ }^{{ }^{\beta_{s}}-1}$ will have the same indices in each pair when $i>\beta_{s}$, for then the blocks are entirely zeros. However block $\epsilon_{\beta} E_{\beta_{s}} J_{\beta_{s}}{ }^{{ }^{\beta_{s}}-1}$ has index $\sigma_{s}$ in one pair and $\sigma_{s}^{\prime} \neq \sigma_{s}$ in the other.

Since the index of block $\epsilon_{i} E_{i}\left[\left(\lambda_{i}-\lambda\right) J_{i}{ }^{0}+J_{i}\right]^{n}$ for $\lambda_{i} \neq \lambda$ depends on the sign of $\epsilon_{i}\left(\lambda_{i}-\lambda\right)^{n}$, it is the same for any even $n$, and the same for any odd $n$. Consider any general set of elementary divisors, and call $\lambda_{1}$ a root such that the $\sigma_{i}$ connected with the set of elementary divisors involving $\lambda_{1}$ are different in the two pairs. Choose $\lambda=\lambda_{1}$ and choose $n$ as above so that the total indices of the corresponding blocks of the two pairs are different. If the remaining blocks have the same total indices for each pair for this $\lambda$ and $n$, we are done. If not, increase $n$ by 2 , whence the difference of the indices of blocks involving $\lambda_{1}$ disappears since the blocks causing the difference become entirely zeros, but the difference of the indices of blocks not involving $\lambda_{1}$ remains unchanged.

This proof shows that condition (2) in the above theorem could be replaced by the following condition $\left(2^{\prime}\right)$ which is more easily applied but less easily stated:

$\left(2^{\prime}\right)$ the matrices $B\left(B^{-1} A-\lambda I\right)^{n}$ and $D\left(D^{-1} C-\lambda I\right)^{n}$ have the same index for all positive integral $n$ which are less than or equal to the order of the matrices involved and of the form $e_{i} \pm 1$, where the $e_{i}$ are the exponents of the real elementary divisors of the pairs, and for all $\lambda$ which are real roots $\lambda_{i}$ involved in these elementary divisors.

UNIVERSITY OF WISCONSIN, Madison, Wis. 